\title{
Collectif d'auteur(e)s
}

Pierre Beaulne, Bernard Élie, Louis Gill, Josée Lamoureux, Marc Lavoie, Sylvie Morel, Victor Palaez, Cécile Sabourin, Vincent van Schendel, Philippe Hurteau, Harvey Mead et al.

(février 2010)

\section{Pour une autre lecture des finances publiques. \\ UNE RÉPONSE AU COMITÉ CONSULTATIF SUR L’ÉCONOMIE ET LES FINANCES PUBLIQUES}

Un document produit en version numérique par Jean-Marie Tremblay, bénévole, professeur de sociologie au Cégep de Chicoutimi

Courriel: jean-marie tremblay@uqac.ca

Site web pédagogique : http://www.uqac.ca/jmt-sociologue/

Dans le cadre de: "Les classiques des sciences sociales" Une bibliothèque numérique fondée et dirigée par Jean-Marie Tremblay, professeur de sociologie au Cégep de Chicoutimi Site web: http://classiques.uqac.ca/

Une collection développée en collaboration avec la Bibliothèque Paul-Émile-Boulet de l'Université du Québec à Chicoutimi

Site web: http://bibliotheque.uqac.ca/ 


\section{Politique d'utilisation de la bibliothèque des Classiques}

Toute reproduction et rediffusion de nos fichiers est interdite, même avec la mention de leur provenance, sans l'autorisation formelle, écrite, du fondateur des Classiques des sciences sociales, Jean-Marie Tremblay, sociologue.

Les fichiers des Classiques des sciences sociales ne peuvent sans autorisation formelle:

- être hébergés (en fichier ou page web, en totalité ou en partie) sur un serveur autre que celui des Classiques.

- servir de base de travail à un autre fichier modifié ensuite par tout autre moyen (couleur, police, mise en page, extraits, support, etc...),

Les fichiers (.html, .doc, .pdf, .rtf, .jpg, .gif) disponibles sur le site Les Classiques des sciences sociales sont la propriété des Classiques des sciences sociales, un organisme à but non lucratif composé exclusivement de bénévoles.

Ils sont disponibles pour une utilisation intellectuelle et personnelle et, en aucun cas, commerciale. Toute utilisation à des fins commerciales des fichiers sur ce site est strictement interdite et toute rediffusion est également strictement interdite.

L'accès à notre travail est libre et gratuit à tous les utilisateurs. C'est notre mission.

Jean-Marie Tremblay, sociologue

Fondateur et Président-directeur général, LES CLASSIQUES DES SCIENCES SOCIALES. 
Cette édition électronique a été réalisée par Jean-Marie Tremblay, bénévole, professeur de sociologie au Cégep de Chicoutimi à partir de :

Collectif d'auteur(e)s : Pierre Beaulne, Bernard Élie, Louis Gill, Josée Lamoureux, Marc Lavoie, Sylvie Morel, Victor Palaez, Cécile Sabourin, Vincent van Schendel, Philippe Hurteau, Harvey Mead et al.

POUR UNE AUTRE LECTURE DES FINANCES PUBLIQUES.

UNE RÉPONSE AU COMITÉ CONSULTATIF SUR L'ÉCONOMIE ET LES FINANCES PUBLIQUES.

Montréal : février 2010, 15 pp.

Texte disponible sur ÉCONOMIE autrement.org.

[Autorisation formelle accordée par Bernard Élie le 16 février 2010 de diffuser ce texte dans Les Classiques des sciences sociales.]

Courriel : elie.bernard@uqam.ca

Polices de caractères utilisée :

Pour le texte: Times New Roman, 12 points.

Pour les citations : Times New Roman, 12 points.

Pour les notes de bas de page : Times New Roman, 12 points.

Édition électronique réalisée avec le traitement de textes Microsoft Word 2008 pour Macintosh.

Mise en page sur papier format : LETTRE (US letter), 8.5'’ x 11'’)

Édition numérique réalisée le 24 février 2010 à Chicoutimi, Ville de Saguenay, province de Québec, Canada. 


\section{ECONOMIEautrement.org}

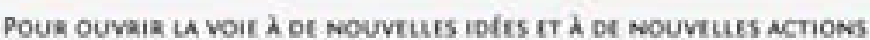

Collectif d'auteur(e)s : Pierre Beaulne, Bernard Élie, Louis Gill, Josée Lamoureux, Marc Lavoie, Sylvie Morel, Victor Palaez, Cécile Sabourin, Vincent van Schendel, Philippe Hurteau, Harvey Mead et al.

“POUR UNE AUTRE LECTURE DES FINANCES PUBLIQUES. UNE RÉPONSE AU COMITÉ CONSULTATIF SUR L'ÉCONOMIE ET LES FINANCES PUBLIQUES”. 1

Montréal : février 2010, 15 pp.

Texte disponible sur ÉCONOMIE autrement.org.

\section{Introduction}

\section{$\underline{\text { Retour à la table des matières }}$}

Nous, économistes impliqués dans différents milieux de la société, affirmons que, tel qu'engagé par le gouvernement québécois, le débat concernant les défis pour l'avenir du Québec est en train de dériver, orienté à partir d'une vision étroite et biaisée des questions économiques, en particulier celle des finances publiques et par une absence de prise en compte d'autres enjeux fondamentaux, comme l'écologie. En tant qu'économistes, nous souhaitons que notre discipline serve véritablement à éclairer les choix de notre société.

C'est pourquoi nous tenons à intervenir pour rectifier les faits, dissiper les fausses interprétations, dégonfler les mythes véhiculés et replacer les questions touchant les finances publiques québécoises dans une autre perspective. Par cette initiative, nous espérons relancer la réflexion sur de nouvelles bases et offrir, à

1 Gouvernement du Québec. Décembre 2009 et janvier 2010. Le Québec face à ses défis - Fascicule 1 et Fascicule 2 dans le texte identifiés par I et II respectivement. 
celles et ceux qui ont à coeur de continuer à faire progresser notre société, une lecture plus exacte des finances publiques que celle qui est actuellement véhiculée sur la place publique ${ }^{2}$.

\section{Le diagnostic des experts du gouvernement, alarmiste et irrecevable}

Le diagnostic posé par les membres du Comité consultatif sur l'économie et les finances publiques constitué par le ministre des Finances, dans leurs deux premiers fascicules, se veut percutant.

«Les choix auxquels fait référence le comité devraient viser : un niveau de dépenses publiques respectant notre capacité de payer ; une fiscalité efficace, ne compromettant pas notre développement économique ; une dette sous contrôle, dont l'importance n'hypothèque pas les choix de nos enfants » (I-p.4 et p.50).

«Cette situation peut se résumer très simplement: nous dépensons plus que nous gagnons et, en conséquence, nous nous endettons [...]» (I-p.7).

En un mot, nous vivons au-dessus de nos moyens, selon les auteurs du Comité. Si la population québécoise se limitait aux mêmes services publics que celle de l’Ontario, les dépenses de l’État seraient réduites de 17,5 milliards de dollars (Ip.11 et suivantes). Nous aurions ainsi un surplus budgétaire et les moyens d'abaisser la dette publique.

La question fondamentale est justement de savoir si les Québécois et les Québécoises acceptent ce diagnostic et veulent troquer les services qu’ils reçoivent et leurs programmes sociaux (assurances automobile, parentale et médicaments, garderies à $7 \$$, faibles frais de scolarité, etc.) contre une fiscalité « efficace et concurrentielle » qui les forcerait soit à mettre la main dans leur poche à répétition pour payer ces mêmes services soit à se priver de ces services. Car il ne faut pas se méprendre : en suivant le diagnostic du Comité consultatif, les services qu’on

2 Les personnes intéressées à approfondir les sujets abordés ici sont invitées à se référer au Dossier finances publiques (http://www.economieautrement.org) qui rassemble plusieurs documents de spécialistes. 
se donne collectivement devraient être assumés de manière privée, comme ailleurs en Amérique du Nord, et cela à un coût généralement supérieur. Un grand nombre de nos concitoyens et concitoyennes moins fortunés n’auraient alors plus accès à de nombreux services. Mais les Québécois et les Québécoises ont choisi d'avoir plus de services que leurs voisins. La mesure de nos défis doit reposer sur les choix de société que nous avons faits, pas sur des comparaisons comptables avec des sociétés différentes qui n’ont pas fait les mêmes choix, à moins de vouloir calquer la société québécoise sur celle de nos voisins. S’appuyant sur une recherche de Luc Godbout, membre du Comité consultatif, et de Suzie St-Cerny, Jacques Parizeau montre bien que le Québec n’est pas l'enfer fiscal que certains décrivent ${ }^{3}$.

Malgré les prétentions du Comité consultatif du gouvernement, le Québec ne s’offre pas la lune. Il est vrai que le Québec dépense plus qu'ailleurs pour les programmes sociaux, notamment pour tout ce qui a trait au soutien à la famille. Cependant, le rendement de ces investissements est assez remarquable quand on constate la remontée du taux de natalité et la progression du taux d'activité des femmes sur le marché du travail depuis l'entrée en vigueur partielle des dispositions de la politique familiale québécoise de 1997.

\section{Sommes-nous trop taxés?}

Le Comité consultatif s’étend en long et en large sur le niveau d'imposition au Québec pour démontrer que le Québec prélève davantage d'impôts et de taxes et qu'il est davantage endetté que les autres provinces.

Nous avons procédé à une comparaison interprovinciale du poids des impôts. Le Québec arrive au premier rang parmi les provinces canadiennes en 2008 pour ce qui est du poids de ces impôts par rapport au revenu personnel, soit 23,5\%. Mais la moyenne canadienne se situe à $22,5 \%$, ce qui n’est pas très éloigné. De manière surprenante, l'Alberta suit de près le Québec, avec des prélèvements fiscaux de 23\% du revenu personnel. L’Ontario n'est pas très loin avec 22,8\%. Cet

3 Parizeau, Jacques. 2009. La souveraineté du Québec, hier, aujourd'hui et demain. Montréal : Les éditions Michel Brûlé, p.216. 
écart de 0,7\% du revenu personnel entre les prélèvements du Québec et ceux de l'Ontario correspond, globalement, à 1,8 milliard de dollars. Ce n’est pas énorme. S’il faut croire le Comité consultatif du gouvernement, le Québec a moins recours à la tarification que les autres provinces. Alors, à moins que les taxes de vente et autres taxes sur les produits affectent beaucoup le tableau, on ne peut pas conclure que les Québécois et Québécoises supportent un fardeau fiscal disproportionné.

Impôts sur le revenu et cotisations sociales

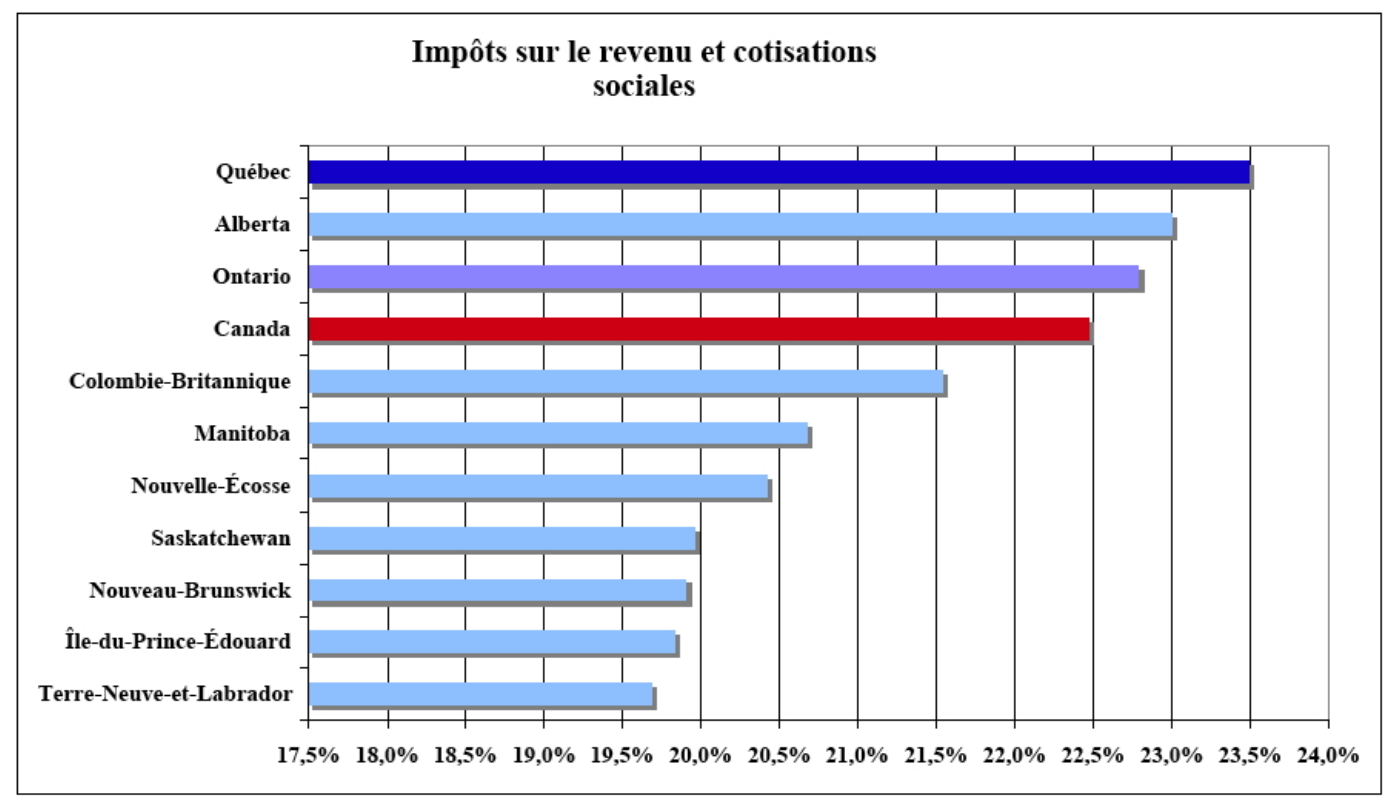

Note : selon le système des comptes nationaux, la différence entre le revenu personnel et le revenu personnel disponible est constituée des impôts directs des particuliers, des cotisations aux régimes d'assurance sociale, ainsi que d'autres transferts, principalement les permis pour véhicules automobiles et les primes d'assurance hospitalisation et de santé, et cela tant au niveau fédéral qu'au niveau provincial.Statistique Canada, Comptes économiques des provinces et des territoires, 


\section{Sommes-nous trop endettés ?}

Au cours de la dernière décennie, le poids de la dette dans l’économie a constamment diminué, bien que le phénomène ait été occulté par les nombreuses réformes de la comptabilité du gouvernement. Entre-temps, le service de la dette est demeuré stable, oscillant entre 7 et 8 milliards de dollars. En proportion des revenus de l'État, le coût du service de la dette, par dollar de recettes, a chuté de 17,7 cents en 1997 à 10,6 cents en 2009. C'est une marge de 4,9 milliards qui s'est dégagée de ce fait.

L’observation est souvent faite qu'il faudrait ajouter à la dette québécoise la quotepart de la dette fédérale, soit environ 100 milliards, ce qui situerait le Québec parmi les pays les plus endettés de l'OCDE. Ceux qui avancent ce point de vue ne prennent jamais en considération les actifs du gouvernement fédéral, dont le Québec devrait aussi recevoir sa quote-part. En tenant compte de cela et en effectuant les calculs sur la même base méthodologique que l'OCDE, le professeur Louis Gill a estimé que la dette nette des administrations publiques du Québec s'établit à 40\% du PIB en 2009, nettement sous la moyenne de l'OCDE qui est de $51 \%$ en 2009 et qui doit atteindre $60 \%$ en 201045 .

Les auteurs des fascicules prétendent que les deux tiers de la dette brute de 151 milliards en 2009 représentent de la « mauvaise dette », c’est-à-dire des dettes contractées pour payer les dépenses courantes, dites aussi « dépenses d’épicerie ». Une telle affirmation ne tient pas. Il s'agit d'une autre fabulation de comptable destinée à culpabiliser les citoyens et les citoyennes en leur faisant croire qu'ils laisseront en héritage la facture de leur comportement désinvolte et irresponsable. De 1998 à 2008, le surplus budgétaire consolidé du gouvernement s’élève à 580 millions. Ce bilan aurait été plus reluisant, d'environ 1 milliard, n’eût été des frasques de la Société générale de financement (SGF) dans ses placements spéculatifs dans les technologies. Entre-temps la dette brute a augmenté de 50 milliards.

4 Gill, Louis. Lettre au ministre des Finances, Raymond Bachand, 29 janvier 2010. Disponible sur le site www.economieautrement.org.

5 Parizeau, Jacques. 2009. op. cit. chapitre VII. 
Cette augmentation s'explique par les effets de la réforme comptable de 2007 et les investissements du gouvernement, et non par les déficits budgétaires, puisqu'il n’y en a pas eu ou presque. Pour ce qui est de la période antérieure à 1997, près de la moitié des déficits annuels découlent des investissements du gouvernement, qui étaient alors comptabilisés dans les dépenses budgétaires. En fin de compte, il faudrait renverser le constat pour conclure que près des deux tiers de la croissance de la dette s'expliquent par les investissements du gouvernement.

Ce qu'il faut souligner, c’est la quantité et la qualité des infrastructures matérielles et des ressources humaines que le Québec moderne s’est données grâce aux investissements de l'État depuis la Révolution tranquille. C’est cela qui sera laissé en héritage. Ceux qui s’inquiètent de l'ampleur de la dette publique pourront se rassurer en examinant les données des Comptes du bilan national dressé par Statistique Canada. (13-022-X). En 2008, la valeur marchande nette des particuliers au Canada, c'est-à-dire après soustraction de leurs dettes, atteignait 5525 milliards. En supposant qu'environ le cinquième de cette somme revient aux Québécois, cela signifie que les actifs financiers et non financiers nets des Québécois représentent environ 7 à 8 fois la valeur de la dette publique. Nous considérons que ceci représente une situation tout à fait acceptable.

\section{Des choix de sociétés à préserver}

La réduction des impôts n’est pas un objectif en soi, si elle conduit à aller contre nos préférences. Ce sont les revenus de l'État qui sont trop bas et non les dépenses qui sont trop élevées. La fiscalité doit s’ajuster à nos choix. Les Québécois et Québécoises ont donné plus d'importance à l'État pour marquer leur volonté d'un partage de la richesse plus égalitaire qu'ailleurs en Amérique du Nord.

Le gouvernement a lui-même contribué activement à creuser un trou dans les finances publiques avec les allègements fiscaux consentis aux particuliers et aux entreprises à la veille des élections de l'automne 2007. Il a notamment remis en baisses d'impôts aux particuliers quelque 700 millions de dollars provenant du règlement du déséquilibre fiscal avec le gouvernement fédéral. Cet argent était destiné à l'amélioration des services. Le dernier budget indique que, pour l'année 
2010, le gouvernement se verra privé de 2,5 milliards de dollars, dont 1,5 milliard découlant des réductions de l'impôt des particuliers dans les budgets antérieurs, et 1 milliard découlant des réductions d'impôts aux entreprises ${ }^{6}$. C’est la moitié du déficit actuel qui s'explique par ces mesures discrétionnaires, le reste étant attribuable aux effets de la récession.

Le gouvernement du Québec a aussi refusé d’utiliser les points de la TPS abandonnés par le gouvernement fédéral, se privant ainsi de recettes nécessaires au financement de nos programmes. Ces derniers gains auraient pu être accompagnés de mesures limitant les effets régressifs de cette taxe à la consommation. La crise dont nous sortons peut-être et la chute des revenus fiscaux qui en a suivi ont grevé les budgets de nos gouvernements. Le gouvernement souhaite un retour à l'équilibre budgétaire en 2013-2014, un délai très court dans le contexte actuel, simple souhait comptable sans véritable vision économique et sociale. Le gouvernement du Québec a donc créé les conditions pour devoir sabrer dans les dépenses et a ensuite mandaté son comité pour y voir. «Qui veut noyer son chien l'accuse de la rage » (Molière, Les femmes savantes, acte II, scène 5).

« Dresser les actions à privilégier et la marche à suivre pour permettre le retour à l'équilibre budgétaire prévu en 2013-2014 et assurer un renforcement des finances publiques à moyen terme [...]» (I-p.6).

La démarche dans laquelle nous enferme le mandat donné par le Ministre au Comité consultatif, en ignorant la dimension structurelle de la crise, nous conduit à fragiliser encore plus les couches de la population les plus vulnérables. Les femmes en premier lieu, dont l'intégration professionnelle de qualité est loin d'être accomplie. Il faut élargir le débat en débordant la question des finances publiques pour discuter des pistes de sortie de crise. C'est dans cette perspective qu'on se serait attendu que le gouvernement pose le problème.

Il faut inverser le raisonnement et, partir des services que les Québécois veulent se donner. Cela implique d’ajuster les revenus aux besoins révélés. Il y a urgence à réhabiliter la dépense publique, non comme un coût, mais comme un levier de développement économique. L’impôt sur le revenu des particuliers doit à nouveau être considéré comme une source légitime de revenus pour l’État et un outil important de réduction des inégalités sociales.

6 Finances Québec. Plan budgétaire 2009-2010, p. C.17. 


\section{Des mythes en série}

De nombreux constats ou conclusions du Comité consultatif, énoncés comme vérités incontestables, représentent autant de mythes répétés à satiété.

Le premier exemple de ce type d'énoncés nous est fourni par l'affirmation selon laquelle ce qui n’est pas bon pour un ménage (l'endettement) est nécessairement mauvais pour une nation ( Nous vivons une situation que nous n’accepterions pas sur le plan individuel », I-p. 7). Cette idée reçue, qui consiste à penser que la logique d'un grand ensemble, comme une société, est identique à celle de ses composantes, c'est-à-dire les individus, repose sur un raisonnement fallacieux. En effet, on ne peut assimiler la dette des administrations publiques aux dettes que contractent des ménages qui dépensent plus qu’ils ne gagnent. D’une part, les dépenses publiques constituent aussi des revenus pour l'ensemble de l'économie ; d'autre part, un gouvernement peut aussi décider de relever ses revenus quand il le veut, ce que ne peut pas toujours faire un individu ou un ménage.

Autre affirmation erronée, la dette actuelle ne serait qu'un poids pour l'économie et un fardeau pour les générations futures. C’est oublier qu'en contrepartie de la dette, nous laissons aussi des actifs : des actifs matériels d abord (infrastructures publiques), mais aussi des actifs non matériels, tels une population éduquée et en santé, des services publics de qualité, une solidarité entre les catégories de la population et un tissu social fort. Autrement dit, au lieu de se demander seulement qui paiera la dette, il faudrait également se demander qui en profitera et quel serait le coût de s'en passer. Ce sont les dépenses publiques qui ont été allouées aux immobilisations, aux services d'éducation et de santé ou encore aux politiques familiales - et la liste est loin d'être exhaustive - qui, en tant qu'investissements productifs, ont été et sont garantes du développement économique. Les dépenses dites «sociales », qui ont de nombreuses implications économiques, ne sont pas seulement un coût : elles sont aussi un investissement pour l’ensemble de la société et son activité productrice. 
Troisième mythe: la ponction inutile par l'État. Les membres du Comité consultatif sur l'économie et les finances publiques, dans leur deuxième fascicule, n’ont pas peur de lancer une affirmation étonnante : « Dès que l’État prélève un dollar, c'est un dollar de moins à la disposition des citoyens ou des entreprises pour dépenser, épargner ou investir ». Comme si l’État ne dépensait pas, n’épargnait pas ou n’investissait pas ! De plus, ils en rajoutent en concluant que « les taxes, impôts et tarifs ont ainsi un impact négatif à long terme sur la croissance économique » (II-p.69). Une telle affirmation suscite de vives controverses entre les économistes, et constitue, pour certains, un plaidoyer pour un désengagement public. À ce compte-là, à quand la disparition de l'État et des services publics?

Le secteur privé serait plus efficace que le secteur public, encore un mythe. Cela ne repose sur aucune démonstration économique rigoureuse. Il peut exister ici et là des dépenses inutiles dans toute administration, autant dans le privé que dans le public, mais rien ne permet de dire que le secteur public est synonyme d'inefficacité, au contraire. Cette croyance est à la base de l'affirmation du fascicule 2 selon laquelle le secteur de la santé aurait intérêt à s’inspirer du secteur de l'éducation où « la concurrence avec un secteur privé important a également pour effet de stimuler l'innovation et la performance ». Dans le même souffle, les auteurs signalent l'ampleur des problèmes de décrochage scolaire et de sousdiplômation qui affectent le système d’éducation (II-p. 13). Étonnamment, aucun lien n'est établi entre les deux phénomènes. Ils évoquent le cas de la Suède qui a systématisé et généralisé la coexistence d’écoles publiques et privées. Or, les analyses crédibles de ce système montrent qu'il s'enfonce depuis les années 1990 dans une dynamique d'inégalités et que les élèves provenant de milieux défavorisés reçoivent de moins en moins d'aide. Le programme PISA ${ }^{7}$ indique que la Suède dégringole depuis 2000 dans le classement et que le pays n'a pas une performance des plus enviables. En ce qui a trait au système de santé, ils citent les Pays-Bas comme exemple de ce qu'il est possible de faire en s'appuyant sur la « concurrence encadrée ». Dans ce pays où tout le monde est tenu d'avoir une assurancesanté privée, l'argumentaire des experts du gouvernement omet une

7 PISA, Programme international pour le suivi des acquis des élèves : une évaluation de nature internationale parmi les pays de l'OCDE. 
question cruciale : qui profiterait de ce type d'évolution, si ce n’est le secteur des assurances commerciales?

Enfin, un mythe des plus tenaces et des plus nuisibles pour la qualité du débat démocratique sur les finances publiques est celui de la neutralité des économistes. Il faut savoir en effet que l'économie est traversée par des oppositions de fond, et non de détail, entre les économistes et qu'elle n'est pas une science neutre. Les économistes que l'on a mobilisés pour produire les fascicules supposés nous offrir un diagnostic indiscutable, livrent une analyse typique de la pensée dominante en économie et présentent une prise de position qui est tout sauf neutre.

\section{L’équité et l’efficacité}

L’hypothèque qui pèse sur les générations futures n'est donc pas la dette. Une véritable hypothèque serait de laisser des services publics de faible qualité. L'exemple le plus significatif de cela est l'éducation. Il faut favoriser la formation, source d'innovation et de productivité à l’origine de la croissance économique. Préserver et améliorer un système d’éducation est un des moyens de permettre à la fois l'efficacité de l'économie et l'équité envers les générations futures. L'abandon scolaire massif que nous connaissons depuis des années est un scandale et un gaspillage pour l'avenir économique et social du Québec. Revaloriser les filières professionnelles et techniques, développer la formation continue et favoriser l'accès aux études post-secondaires doit être une priorité. Cependant, l'équité intergénérationnelle n’est pas la seule équité qui soit compromise par le décrochage scolaire. L’équité intra-générationnelle l'est également en raison des profondes inégalités qui se creusent aujourd'hui sous nos yeux entre les jeunes euxmêmes. Le développement de trajectoires éducationnelles pour les mieux nantis contribue à accentuer l'iniquité intragénérationnelle. Le décrochage est, en bonne partie, le produit des mauvais choix de politiques budgétaires adoptées par le passé. 
Nous ne saurions sous-estimer également l'importance des autres services publics qui poursuivent aussi des objectifs d'équité ${ }^{8}$. Ceux de la santé, mais aussi ceux de garde d'enfants ou de soutien aux autres personnes dépendantes sont nécessaires pour poursuivre l'équité entre les hommes et les femmes, objectif qui est loin d'être réalisé encore. Les services publics de l'emploi le sont aussi pour assurer, notamment, l'équité entre les personnes en emploi et celles qui en sont privées. Les services aux personnes vieillissantes assurent l'équité intergénérationnelle qu'il nous incombe aujourd'hui de garantir. Il s'agit de la dette que nous devons honorer aujourd'hui envers nos aînés et aînées pour l’héritage qu'ils nous ont légué en construisant, par leur contribution productive passée, la société québécoise.

L’équité et l'efficacité sont liées : une société inéquitable n’est pas une société efficace, puisqu'elle ne permet pas à sa population de réaliser son potentiel.

\section{La dette écologique : un problème incontournable pour le développement futur}

Nous sommes d'avis que la pire hypothèque que nous léguons actuellement aux générations futures est une planète pillée et polluée. Cette dette écologique pose la nécessité d’un véritable développement durable qui n’a rien à voir avec la définition qu’en donne le Comité du ministre Bachand. Les auteurs réduisent cette obligation à une question budgétaire, suggérant que son souci est de « faire bénéficier le Québec de finances publiques équilibrées à long terme, respectant la capacité des générations futures d’effectuer leurs propres choix » et les auteurs prétendent que ceci « correspond à la définition même du développement durable » (I-p.4). Cette position particulière du Comité consultatif est plus réductrice que celle du Premier ministre.

8 Mackenzie, Hugh et Richard Shillington. 2009. L'aubaine discrète du Cana$d a$, les avantages tirés des services publics. Centre canadien de politiques alternatives, avril. 
« La crise économique n'est pas le seul défi auquel nous faisons face. Cette économie, que tous les pays du monde essaient aujourd'hui de relancer, a fait une si mauvaise utilisation des ressources de la planète qu'elle est devenue une menace. Et il y a aussi la crise environnementale. J'ai la profonde conviction que cette double crise déclenchera la véritable révolution du développement durable. Un meilleur monde en émergera, et le Québec y contribuera ${ }^{9}$.

En dépit de l’urgence soulevée par le Premier ministre, à Copenhague le Québec présentait une position respectant à peine la moitié de la cible jugée minimale par la communauté scientifique ${ }^{10}$, sous prétexte qu'il ne pourrait pas supporter une dette plus importante. Malgré tout, le Québec se dit intéressé par un marché du carbone pour gérer le problème des changements climatiques. Cependant s’engager dans un tel marché comporterait des obligations financières de plusieurs milliards de dollars de fonds publics dans un proche avenir.

Le comité consultatif ne parle pas de cette dette écologique. Son mandat est trop réducteur. En plus des coûts des changements climatiques, on pourrait inclure ceux qui proviennent de l'exploitation irresponsable de nos ressources naturelles (forêts, pêches, mines et agriculture). La croissance économique dont parle le mandat du Comité consultatif n’est vraiment pas en accord avec les préoccupations de la société québécoise d’aujourd’hui.

\section{D’autres sources de revenus pour l’État}

Malgré tout, il faut bien que l'État se finance. Le Comité du Ministre favorise alors des hausses de la tarification de préférence à l'augmentation des impôts sur les revenus. En effet, selon les membres du comité, l’impôt sur les revenus provoquerait des effets plus pervers que la tarification. L'impôt serait une taxe sur le travail et ainsi « on diminue l'incitation à travailler et on décourage les travailleurs qui le désirent à ajouter à leur prestation de travail » (II-p.70). Les récentes

9 «L'occasion de se démarquer », Discours inaugural du Premier ministre à l’ouverture de l'Assemblée nationale le 10 mars 2009, p.7, http://www.plq.org/pdf/discours_inaugural.pdf.

10 Quelle cible de réduction d'émissions de gaz à effet de serre à l'horizon 2020 ?, MDDEP, octobre 2009, p.21 et suivantes. 
réductions d'impôt du gouvernement Charest ont-elles contribué à l'augmentation des prestations de travail pour ceux et celles qui en ont bénéficié ? Où sont les évaluations démontrant que les quelques dizaines de dollars de moins payées par certains contribuables ont modifié vraiment le comportement des Québécois et Québécoises ? Devant une telle affirmation, le Comité consultatif privilégie donc l'augmentation des tarifs, affirmant que «la tarification est une forme de prélèvement moins dommageable pour l'activité économique » (II-p.77). Une plus grande importance accordée à la tarification se justifie au Québec, selon eux, parce que nous l'utilisons moins qu'ailleurs ! Mais surtout, soutiennent-ils, l'utilisation plus importante des tarifs « induit les bons comportements de la part des utilisateurs de services publics, en établissant un lien direct et transparent entre l'utilisation du service et son coût pour celui qui en bénéficie » (II-p.81). Les prélèvements fiscaux de l'État ne sont analysés que dans une perspective individuelle, d'utilisateur-payeur, sans tenir compte de la répartition de la richesse et de la réduction des inégalités.

Comme il y a plusieurs manières d'augmenter les prélèvements, les principes qui doivent nous guider à cet égard sont ceux de l'équité et de la protection de l'environnement. L'ampleur des hausses de contributions fiscales doit être en rapport avec la capacité de payer des entreprises ou des particuliers. En ce sens, il faut rétablir une structure plus progressive des taux d'imposition. Certaines tarifications et taxes comme celles des produits énergivores ou de luxe devraient être haussées. N'oublions pas non plus l'absence de véritables redevances sur les ressources naturelles que, lorsqu'elles existent, le Québec oublie, semble-t-il, de réclamer.

L'objectif du gouvernement du Québec de réduire de 4,6\% à 3,2\% le taux annuel de croissance des dépenses de programmes d'ici 2013-2014, signifie une compression des dépenses de 900 millions de dollars par année, de près de 4 milliards en 4 ans. Est-ce souhaitable ? Déjà, la politique de réduction de la fonction publique (ne combler qu'un départ à la retraite sur deux) s'est révélée catastrophique. La présidente du Conseil du trésor Monique Gagnon-Tremblay a même annoncé sa révision. Dans une perspective de renouvellement du personnel, la dégradation des conditions de travail qui prévaut depuis plusieurs années dans le secteur public ne peut que rendre très difficile le recrutement de ressources rares, également en demande dans le secteur privé. Avoir des services publics de qualité 
aptes à répondre aux besoins des Québécois et des Québécoises et à leurs entreprises ne peut se faire à rabais, aujourd'hui comme dans le futur. De plus, la « reprise », depuis le second trimestre de 2009, est très fragile et tout retour précipité à l’équilibre budgétaire ne pourrait que la compromettre.

\section{Conclusion}

Les propos avancés dans les deux premiers fascicules du Comité consultatif sur les finances publiques sont superficiels, tendancieux et alarmistes. Ils reprennent, comme s’il s’agissait de vérités universelles, plusieurs mythes. À partir d'un diagnostic déformé, les solutions qui s’ensuivent, présentées de manière plus détaillée dans le dernier fascicule, surprennent peu quand on connaît la composition du comité : coupures budgétaires, recours accrû à la tarification des services publics, privatisation et mise en concurrence des services publics avec les services privés comme étant les seules options possibles. Pendant ce temps, on occulte les vraies questions. Quels sont les défis économiques des années à venir ? Quels moyens souhaitons-nous adopter, comme société, pour les relever ? Quelles ressources voulons-nous y consacrer et par quels moyens de financement les constituer ? De toute évidence, le débat reste ouvert. Il faudra reprendre l'exercice sérieusement.

\section{liste des économistes signataires au 20 février 2010}

Le groupe de rédaction :

Pierre Beaulne (CSQ), Bernard Élie (UQAM), Louis Gill (UQAM), Josée Lamoureux (CSN), Marc Lavoie (U. d'Ottawa), Sylvie Morel (U. Laval),
Victor Pelaez (Économie autrement), Cécile Sabourin (UQAT) Vincent van Schendel (UQAM) Avec la collaboration de : Philippe Hurteau (Institut de recherche et d'information socioéconomique - IRIS) Harvey Mead (Nature Québec). 
Et :

Omar Aktouf (HEC), François Aubry (CSN), Monique Audet (FTQ), Brian Barton (UQTR), Kamel Béji (U. Laval), Gilles Bourque (Éditions vie économique),

Maurice Carel (U. Laval),

Marie-Thérèse Chicha (U. de Montréal),

Harold Chorney (Concordia), André-Pierre Contandriopoulos (U. de Montréal),

Lise Côté (FTQ),

Roland Côté (UQAM),

Guy Debailleul (U. Laval), Christian Deblock (UQAM), Daniel Desjardins (CEGEP Maisonneuve),

Gilles Dostaler (UQAM), Marc-André Gagnon, Jean-Jacques Gislain (U. Laval), Frédéric Hanin (U. Laval), Pierre-Antoine Harvey,
Pierre J. Hamel (INRS),

Pierre-André Julien (UQTR Institut des PME),

Pierre Laliberté (FTQ),

Richard Langlois (CSQ),

Robert Laplante (Institut de recherche en économie contemporaine IREC),

France Laurendeau (FTQ),

Raymonde Leblanc, Michel Lizée (UQAM), Margie Mendell (U. Concordia), Jacques Mercier (U. Laval),

Pierre Paquette (Collège militaire de St-Jean),

Éric Pineault (UQAM), Lise Pomerleau (CSN), Jean-Pierre Racette (Société d'habitation populaire de l'Est de Montréal),

Claude Rioux (CSN), Ruth Rose (UQAM), Mario Seccareccia (U. d'Ottawa), Diane G. Tremblay (TELUQ), Sylvie Vachon (CSN), Louise Vandelac (UQAM),

\section{Fin du texte}

